\title{
Deformations and Tensile Fracture of Carbon Nanotubes Based on the Numerical Homogenization
}

\author{
M. CHWA * \\ Cracow University of Technology, Institute of Machine Design, 31-864 Cracow, Poland
}

\begin{abstract}
The existence of defects in nanostructures has a significant influence on their mechanical properties. A nonlinear finite element model of single-walled carbon nanotube without and with atom vacancy defects is presented. The numerical efficient formulation for carbon nanotubes is discussed considering the geometry, together with a finite element discretization, including the atomic potential. The effective mechanical properties are evaluated based on the homogenization theory. The results for pristine and defective single-walled carbon nanotube are presented in the form of stress-strain curves. Vacancy defects noticeably reduce the failure stresses and failure strains.
\end{abstract}

DOI: 10.12693/APhysPolA.131.440

PACS/topics: $62.25 .+\mathrm{g}$

\section{Introduction}

The specific properties of carbon nanotubes still attract the attention of scientific world. The costs of experimental measurements and uncertainty of the obtained results demand a support from the theoretical description. For example, only for Young's modulus, the reported values oscillate from 0.68 GPa to $5 \mathrm{TPa}$ (e.g., [1-3]). Besides, theoretical calculations have predicted failure strains and failure stresses of carbon nanotubes much higher than those of the few direct mechanical measurements [4]. The possible explanation takes into account the role of defects in deterioration of the strength peak. Considering their quasi-one-dimensional atomic structure, one can expect that even a small number of defects will result in degradation of their mechanical characteristic. A lot of papers consider the modeling of imperfect carbon nanotubes and the role of vacancy defects in the fracture behavior under axial tension. Mostly, the single- or two-atom vacancies $[5,6]$ are analyzed, however the large defects are also taken into account [7-9].

The present work is focused on the effective mechanical properties of pristine and defective single-walled carbon nanotube. The estimation is based on the molecular mechanics, theory of homogenization and finite element modeling. Here, the carbon nanotube is presented as a space network of beams with a nonlinear behavior, based on the Tersoff-Brenner potential. Only the interactions between the closest neighbors in $\mathrm{C}-\mathrm{C}$ network are considered. In order to simulate an imperfect carbon nanotube, atom vacancies are introduced, which produce a distortion in the perfect configuration of hexagonal arrays. The differences in the effective mechanical properties between pristine and defective $(5,5)$ carbon nanotubes are presented. The present paper is an extension of previous works [10-13].

*e-mail: mchwal@pk.edu.pl

\section{Atomic interaction}

The specific properties of carbon nanotubes are associated with the space arrangement of atomic bonds. The system of atomic bonds in carbon nanotubes is similar to the atomic arrangement in graphite structure, in which, in-plane, the strong covalent bonds are present. As a result, considering the carbon nanotube as a rolledup graphite layer, we have the structure with high stiffness and strength. The description of the nanostructure behavior starts from the consideration of the interatomic potential that is composed of special functions and physical constants based on overall rules of solid state physics. The nature of interactions depends on complicated quantum effects that occur at the level of elementary parts of atom and control chemical properties. In order to obtain reliable results in numerical simulations basing on the solid state physics, the potentials should explain the quantum effects even in the averaging sense. In general, an interatomic potential $V\left(r_{i j}\right)$ describes how the potential energy of a $N$-atom system depends on the coordinates of atoms. It is composed of the outside force field energy, potential energy between atom pairs, potential energy between three atoms and so on. As to make the numerical simulations shorter, the atomic interaction between other than neighboring atoms are omitted.

For carbon atoms, the Tersoff-Brenner potential is commonly used $[14,15]$. This potential describes the short- and long-distance atomic interactions and postulates that the strength of a bond between two atoms $i$ and $j$ is not constant but depends on the local environment. The $\mathrm{C}-\mathrm{C}$ bond becomes weaker through the existence of other bonds that influence the atom $i$. The value of bond weakness depends on the $k$ atom position [12]. In order to be closer to the real interatomic behavior, the TersoffBrenner potential introduces an angle component. For carbon atoms the potential is presented as follows:

$$
V^{\mathrm{TB}}\left(r_{i j}\right)=V_{\mathrm{R}}^{\mathrm{TB}}\left(r_{i j}\right)-\bar{B}_{i j} V_{\mathrm{A}}^{\mathrm{TB}}\left(r_{i j}\right),
$$


where components $V_{\mathrm{R}}^{\mathrm{TB}}$ and $V_{\mathrm{A}}^{\mathrm{TB}}$ describe the repulsive and the attractive parts respectively:

$$
\begin{aligned}
& V_{\mathrm{R}}^{\mathrm{TB}}\left(r_{i j}\right)=f^{\mathrm{TB}}\left(r_{i j}\right) \frac{D_{e}^{\mathrm{TB}}}{S^{\mathrm{TB}}-1} e^{-\sqrt{2 S^{\mathrm{TB}}} \beta^{\mathrm{TB}}\left(r_{i j}-R_{i j}\right)}, \\
& V_{\mathrm{A}}^{\mathrm{TB}}\left(r_{i j}\right)=f^{\mathrm{TB}}\left(r_{i j}\right) \frac{D_{e}^{\mathrm{TB}} S^{\mathrm{TB}}}{S^{\mathrm{TB}}-1} e^{-\sqrt{\frac{2}{S^{\mathrm{TB}}}} \beta^{\mathrm{TB}}\left(r_{i j}-R_{i j}\right)},
\end{aligned}
$$

where $r_{i j}$ is the distance between atoms $i$ and $j, f^{\mathrm{TB}}\left(r_{i j}\right)$ is the cut-off function, $S^{\mathrm{TB}}$ and $\beta^{\mathrm{TB}}$ are constants. The component $B_{i j}$, which defines the order of atomic bonds, is given by:

$$
\begin{aligned}
& B_{i j}=\left[1+\sum_{k(\neq i, j)} G\left(\theta_{i j k}\right)\right]^{-\delta}, \\
& G(\theta)=a_{0}\left[1+\frac{c_{0}^{2}}{d_{0}^{2}}+\frac{c_{0}^{2}}{d_{0}^{2}+(1+\cos \theta)^{2}}\right], \\
& \bar{B}_{i j}=\left(B_{i j}+B_{j i}\right) / 2,
\end{aligned}
$$

where $\theta_{i j k}$ is the angle between bonds $i-j$ and $i-k$. The material data adopted here is as follows [15]: $D_{e}^{\mathrm{TB}}=$ $0.9612 \mathrm{nNnm}, S^{\mathrm{TB}}=1.22, \beta^{\mathrm{TB}}=21 \mathrm{~nm}^{-1}, R_{i j}=$ $0.139 \mathrm{~nm}, \delta=0.5, a_{0}=0.00020813, c_{0}=330, d_{0}=3.5$.

\section{Carbon nanotube numerical model}

The present model of $(5,5)$ carbon nanotube combines the geometry together with a finite element discretization, incorporating the atomistic potential. This approach offers several advantages, mainly related to the computational efficiency and to the possibility of a simple implementation using the existing commercial finite element codes. We base on the assumption that the $\mathrm{C}-\mathrm{C}$ bonds are the $3 \mathrm{D}$ beam elements having the length $L$ and diameter $d$ characterizing a circular cross-sectional area of the elements (Fig. 1a). The connection between the molecular and the continuum mechanics can be made by a suitable definition of $3 \mathrm{D}$ beam mechanical properties.

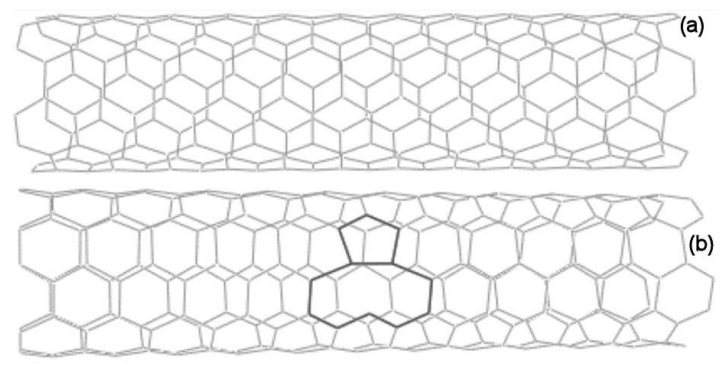

Fig. 1. Numerical model of (a) pristine and (b) defective (one atom vacancy) armchair $(5,5)$ carbon nanotube.

Assuming a round section of the beam element, three stiffness parameters, i.e., the tensile resistance $E_{A}$, the flexural rigidity $E_{I}$ and the torsional stiffness $G_{J}$, need to be determined for the deformation analysis. By considering the energy equivalence between local potential energies in the computational chemistry and the elemental strain energies in the structural mechanics, a direct relationship between the structural mechanics parameters and the molecular mechanics force field constants can be established [16], i.e.:

$$
\frac{E_{i} A_{i}}{r_{i}}=k_{r i}, \quad \frac{E_{i} I_{i}}{r_{i}}=k_{\theta i}, \quad \frac{G_{i} J_{i}}{r_{i}}=k_{t i},
$$

where $k_{r_{i}}, k_{\theta i}$ and $k_{t i}$ are the force field constants in the molecular mechanics.

Let us note that the present formulation is an extension of the models proposed in Refs. 16 and 17. It allows to investigate large deformations of carbon nanotubes since the beam length $L$ in Eq. (4) is replaced by an actual beam length $r_{i}$. Using the interatomic potentials shown in Eqs. (1)-(3), the stretching force that results from the bond elongation $\Delta r_{i}$ and the twisting moment that results from the bond angle variation $\Delta \theta_{i}$, can be computed as follows:

$$
\begin{aligned}
& F\left(\Delta r_{i}\right)=\frac{\partial V}{\partial r_{i}}, \quad M\left(\Delta \theta_{i}\right)=\frac{\partial V}{\partial \theta_{i}}, \\
& \Delta r_{i}=r_{i}-r_{0 i}, \quad \Delta \theta_{i}=\theta_{i}-\theta_{0 i}, \quad i=1,2 .
\end{aligned}
$$

The derivatives in Eq. (5) are expanded in the Taylor series up to the first derivative (linear terms) only. However, the initial values $r_{0 i}$ and $\theta_{0 i}$ are modified at each iteration step since, in fact, both the tensile forces and the twisting moments are nonlinear with respect to the bond length and to the bond angle, respectively. By assuming the circular cross-section of the beam with diameter $d_{i}$, and setting $A_{i}=\pi d^{2} / 4, I_{i}=\pi d^{4} / 64$, Eqs. (4) and (5) give:

$$
d_{i}=4 \sqrt{\frac{k_{\theta i}}{k_{r i}}}, \quad E_{i}=\frac{k_{r i}^{2} r_{i}}{4 \pi k_{\theta i}} .
$$

Then, following the procedure of the finite element structural mechanics technique, the nanotube deformation under certain loading conditions can be readily solved.

Let us model the defective carbon nanotubes as the structures with missing carbon atoms. For example, for the single atom vacancy, the 12-membered ring (two hexagons) is reconstructed to a five-member ring and a nine-member ring (Fig. 1b), and finally we obtain nonaxisymmetric carbon nanotube. Of course, using the similar approach it is possible to model various types of defects [12].

\section{Numerical homogenization}

In the present approach, the Young's modulus of the material is defined as the ratio of the longitudinal stress to the longitudinal strain as obtained from the uniaxial tension test. Following this definition, the homogenized Young's modulus of $(5,5)$ carbon nanotube is calculated using the following equation:

$$
\begin{aligned}
& E_{\text {long }}=\frac{\left\langle\sigma_{\text {long }}\right\rangle}{\left\langle\varepsilon_{\text {long }}\right\rangle}, \quad\left\langle\sigma_{\text {long }}\right\rangle=\sum_{k=1}^{N_{\text {beams }}} \sigma_{\text {long }}^{k}, \\
& \left\langle\varepsilon_{\text {long }}\right\rangle=\sum_{k=1}^{N_{\text {beams }}} \varepsilon_{\text {long }}^{k},
\end{aligned}
$$


where $\left\langle\sigma_{\text {long }}\right\rangle\left(\left\langle\varepsilon_{\text {long }}\right\rangle\right)$ is an average longitudinal stress (strain) component computed as the sum of the longitudinal components of each individual beams $k$, characterizing $\mathrm{C}-\mathrm{C}$ bonds. At each load step, related to the increment of the axial displacement, both molecular force field constants from Eq. (4) and the beam geometrical and mechanical properties from Eq. (6) were computed in order to find the longitudinal stress/strain components for individual beams. This iterative, nonlinear procedure was carried on up to the prescribed end of the deformation process, see [13].

\section{Stress-strain curves}

The calculated stress-strain curves of the pristine and the defective carbon nanotubes from the proposed model are presented in Fig. 2.

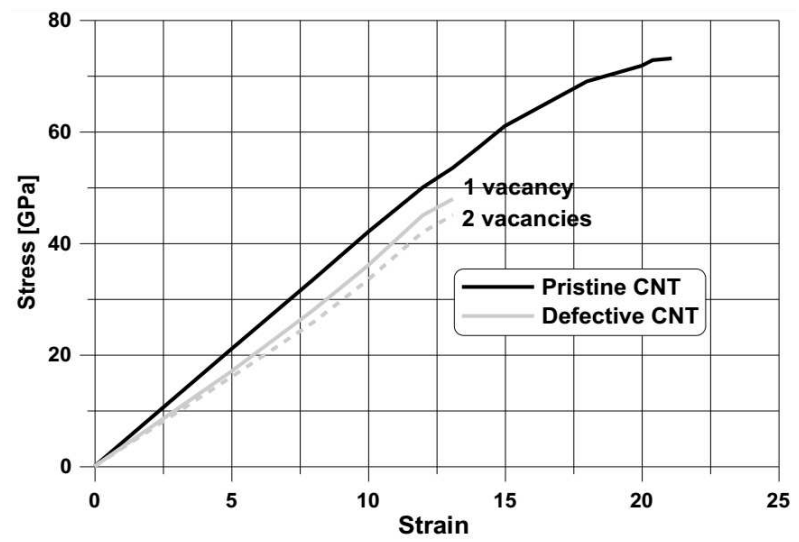

Fig. 2. Tensile stress-strain curves for pristine and defective $(5,5)$ nanotubes.

Initially, we have computed the Young's moduli of $(5,5)$ armchair carbon nanotubes. The predicted initial Young's moduli of nanostructures were $797 \mathrm{GPa}$, $708 \mathrm{GPa}$ and $682 \mathrm{GPa}$ for the pristine and the defective carbon nanotubes with one and two vacancies, respectively, which agrees well with the experimental value and other theoretical descriptions [1]. However, the numerical values were strongly dependent on the form of the assumed interatomic potential and the form of defects. We have observed that the defects clearly reduce the failure stresses by $34 \%$, and failure strains by $38 \%$. They may reduce also the buckling stresses for compressive loads and change the modal characteristic of carbon nanotubes [13].

\section{Conclusions}

It is obvious that the reduction of mechanical properties of carbon nanotubes depends on the assumed type of defects. According to the present results, the evident reduction in failure characteristic is observed. Moreover, the initial properties, including Young's modulus, are deteriorated versus vacancy number. The proposed approach combining the molecular mechanics, the finite element method and the homogenization theory may be applied as the defect identification method for carbon nanotubes. Next, the defects identification can help in prediction of other properties, such as electrical properties, which are particularly sensitive to geometrical modifications.

\section{References}

[1] M.M. Shokrieh, R. Rafiee, Mech. Compos. Mater. 46, 155 (2010).

[2] P.C. Ma, J.K. Kim, Carbon nanotubes for polymer reinforcement, CRC Press, Boca Raton 2011.

[3] M. Chwał, A. Muc, Compos. B 88, 295 (2016).

[4] A. Krishnan, E. Dujardin, T.W. Ebbesen, P.N. Yianilos, M.M.J. Treacy, Phys. Rev. B 58, 14013 (1998).

[5] A.V. Krasheninnikov, K. Nordlund, J. Vac. Sci. Technol. B 20, 728 (2002).

[6] S.L. Mielke, D. Troya, S. Zhang, J.L. Li, S. Xiao, R. Car, R.S. Ruoff, G.C. Schatz, T. Belytschko, Chem. Phys. Lett. 390, 413 (2004).

[7] S. Zhang, S.L. Mielke, R. Khare, D. Troya, R.S. Ruoff, G.C. Schatz, T. Belytschko, Phys. Rev. 71, 115403 (2005).

[8] W.H. Duan, Q. Wang, K.M. Liew, X.Q. He, Carbon 45, 1769 (2007).

[9] K.M. Liew, X.Q. He, C.H. Wong, Acta Mater. 52, 2521 (2004).

[10] M. Chwał, Procedia Eng. 10, 1579 (2011).

[11] M. Chwał, Adv. Material. Res. 849, 88 (2014).

[12] A. Muc, M. Chwał, A. Banaś, Biuletyn WAT 61, 135 (2012).

[13] M. Chwał, Appl. Mech. Material. 477, 1225 (2014).

[14] J. Tersoff, Phys. Rev. Lett. 56, 632 (1986).

[15] D.W. Brenner, Phys. Rev. B 42, 9458 (1990).

[16] K.I. Tserpes, N. Silvestre (Eds.), Modeling of carbon nanotubes, graphene and their composites, Springer, Heidelberg 2014.

[17] C. Li, T.W. Chou, Compos. Sci. Technol. 66, 2409 (2006). 